El empleo y el trabajo como dimensiones de análisis. El proceso de inserción laboral de estudiantes del FinEs2

Federico Martín González

Question, Vol. 1, N. 56, e011, octubre-diciembre 2017. ISSN 1669-6581

http://perio.unlp.edu.ar/ojs/index.php/question/article/view/4382

FPyCS | Universidad Nacional de La Plata

La Plata | Buenos Aires | Argentina

Recibido: 29-09-2017 Aceptado: 17-11-2017

Cita sugerida: González, F. (2017). El empleo y el trabajo como dimensiones de análisis. El proceso de inserción laboral de estudiantes del FinEs2. Question, 1(56), e011. doi: https://doi.org/10.24215/16696581e011

\title{
El empleo y el trabajo como dimensiones de análisis. EI proceso de inserción laboral de estudiantes del FinEs2
}

\author{
The employment and the work like dimensions of analysis. The labor \\ insertion of FinEs2's students
}

Federico Martín González

Laboratorio de Estudios en Sociología y Economía del Trabajo; Instituto de Investigaciones en Humanidades y Ciencias Sociales; Universidad Nacional de La Plata (Argentina) federicomartin.gon@gmail.com

\section{Resumen}

El artículo analiza el proceso de inserción laboral de jóvenes estudiantes del Plan de Finalización de Estudios Secundarios (FinEs2) de un barrio de la Delegación municipal de Melchor Romero. Desde una perspectiva metodológica cualitativa, se analizan las formas en que se configuran las posiciones de los jóvenes en el mercado de trabajo y las disposiciones sobre las distintas esferas del mundo laboral. Finalmente, a partir de la articulación de las dimensiones objetivas -relacionadas a la estabilidad y a las condiciones de trabajo- y subjetivas -vinculadas al reconocimiento material y simbólico- se avanza en la construcción de cuatro tipo 
de posiciones en el mercado de trabajo, que permiten problematizar las relaciones entre nivel de formación, acceso y calidad del empleo.

Palabras clave: educación: empleo; trabajo; inserción laboral; jóvenes.

Abstract

The aim of this paper is to analyze the labor insertion processes of young people who finished high school under the new public education terminality policies, such as the Plan de Finalización de Estudios Secundarios (FinEs2). From a perspective based on qualitative methods, we study labor market's positions and working arrangements. As a result, we advanced in the connections between objective dimensions -associated with stability and working conditions- and subjective dimensions -linked to material and symbolic recognition- to define four types of positions on the labor market which allow to problematize the relationships between the education level, access and quality of employment.

Keywords: education; employment; work; labor insertion; young people.

A pesar de los múltiples debates sobre el mundo del trabajo, el empleo continúa siendo un elemento central en la configuración de las experiencias biográficas. Partiendo de la centralidad de lo laboral, en este artículo se aborda el proceso de inserciones laborales de jóvenes que finalizaron su formación secundaria en el marco del Plan de Finalización de Estudios Secundarios (FinEs2).

El Plan FinEs2 es una política de terminalidad educativa que surge en 2008, en relación directa con los desafíos que la última Ley de Educación Nacional Nro. 26.206 pone en escena. Es decir, la instauración de la obligatoriedad de la educación secundaria promovió, como mínimo, dos procesos importantes. Por un lado, el debate y la necesidad de poner en discusión el formato de la escuela secundaria cuya tradición es caracterizada por la exclusión de jóvenes pertenecientes a orígenes sociales bajos. Por el otro lado, la implementación de distintas políticas públicas de terminalidad educativa, con el objetivo de garantizar la obligatoriedad de dicho nivel a jóvenes y adultos que por distintos motivos no lo finalizaron.

Desde 2008, y con mayor fuerza en 2009, se implementó el Plan FinEs2 en la ciudad de La Plata a partir de la articulación entre la Dirección de Educación de Adultos de la Dirección General de Cultura y Educación de la provincia de Buenos Aires y distintas organizaciones 
sociales con fuerte impronta territorial, en barrios donde el alcance de las escuelas de adultos, denominadas Centros Educativos de Nivel Secundario (CENS), ha sido insuficiente.

El formato del Plan FinEs2 fue concebido, en un primer momento, para completar los estudios de cooperativistas y familiares que no habían finalizado la formación secundaria. De esta forma, dicha política planteó, desde sus comienzos, vinculaciones entre los mundos de la educación y del trabajo a partir de la articulación del Ministerio de Educación y el Programa Ingreso Social con Trabajo, del Ministerio de Desarrollo Social.

Este artículo parte del trabajo de campo realizado con estudiantes de una sede educativa del FinEs2 de un barrio de la Delegación municipal de Melchor Romero, en el marco de una investigación doctoral sobre la construcción de experiencias educativas de estudiantes del Plan FinEs2 durante el periodo 2013-2017. Específicamente, se abordará el interrogante sobre cómo se delinean los procesos de inserción laboral de los jóvenes estudiantes, prestando especial atención a las formas en que se configuran las posiciones en el mercado laboral y las producciones simbólicas en torno al mundo del trabajo.

Para ello se recupera la distinción entre "precariedad del empleo" y "precariedad del trabajo" que realiza Paugam (2000), con el objetivo de avanzar en la articulación de las dimensiones objetivas y subjetivas presentes en las inserciones laborales. Esto posibilitará la definición de tipos de posiciones en el mercado de trabajo y la problematización de las relaciones lineales que se establecen entre nivel de formación, acceso y calidad del empleo.

Como se planteó anteriormente, en este artículo se retoma el análisis de datos construidos en una investigación en curso que, desde un enfoque metodológico cualitativo, combina distintas técnicas: observación participante y no participante, entrevistas en profundidad a jóvenes y adultos estudiantes del FinEs2 y análisis de fuentes secundarias. Se hizo hincapié en el trabajo, con una serie de doce entrevistas en profundidad realizadas durante 2015 a estudiantes hombres y mujeres, donde se indagó sobre la configuración de trayectorias biográficas, prestando especial atención a las dimensiones laborales y educativas.

Por último, el texto se encuentra estructurado en cuatro secciones. En primer lugar, se desarrolla una breve referencia al mercado de trabajo argentino, dando cuenta de las desigualdades que atraviesan a los jóvenes como grupo social. En segundo lugar, se presenta un análisis de las dimensiones objetivas y subjetivas que intervienen en los procesos de inserción laboral de los jóvenes estudiantes. En tercer lugar, se avanza en la articulación de las dimensiones presentadas para la construcción de cuatro tipos de posiciones en el mercado de trabajo. Por último, se exponen algunas líneas de cierre teniendo en cuenta el interrogante planteando.

\section{Mercado de trabajo y desigualdades: el lugar de los jóvenes}


La transición hacia el mundo laboral adquiere importancia en tanto problema sociológico, debido a las grandes transformaciones acontecidas en los modelos societales. La inicial constitución como problema social posibilitó la inserción de esta preocupación en el campo de las ciencias sociales (Jacinto, 2010).

La participación de los jóvenes en el mercado de trabajo está marcada por determinadas características, principalmente la precariedad y el desempleo (Perez, 2008; Battistini y Mauger, 2012). Si bien los jóvenes no constituyen un grupo homogéneo y, por ende, existen diversas trayectorias y experiencias vinculadas al trabajo: la informalidad, la inestabilidad y la flexibilidad se acentúan en dicho sector (Longo, 2011). Es por ello que sostenemos la necesidad de incorporar un andamiaje conceptual alternativo a aquel que concibe a la inserción laboral como el momento en que los jóvenes acceden a una posición estable en el mercado de trabajo. Frente a dicha conceptualización, hablaremos de transiciones e inserciones bajo la idea de procesos de periodos largos de tiempo, con aspectos multidimensionales e interacciones entre trabajo, estudio e inactividad (Jacinto, 2010; Busso, Longo y Pérez, 2011).

En las dinámicas que intervienen en los procesos de inserción laboral influyen múltiples desigualdades. Es así que los itinerarios de los jóvenes en el mundo laboral están cruzados por diversos procesos como la segregación territorial, la discriminación educativa, entre otros (Kessler, 2014). Sin embargo, sostenemos que el origen social es aquella variable central que delinea las otras, a pesar de sus relativas autonomías. Desde esta mirada, recuperamos algunos trabajos que con una perspectiva cuantitativa 0 de articulación de enfoques metodológicos nos permiten trazar un panorama macro del escenario en el que los jóvenes se posicionan. Para este apartado, retomamos la pregunta en torno a cómo el origen social influye en el proceso de configuración de inserciones en el mercado de trabajo.

A partir de la crisis del modelo de acumulación, basado en la valorización financiera canalizada en 2001 y 2002, se gesta la apertura de un nuevo escenario que presenta una de las mayores disputas en torno a la definición de un nuevo patrón de acumulación. Existe un relativo consenso que, a partir de 2003, hay una presencia más activa del Estado en los campos de la política social y redistributiva -anteriormente presente pero bajo modalidades regresivas- y un crecimiento económico que produjo una mejora de indicadores sociales. Sin embargo, las condiciones en el mercado de trabajo no evolucionaron de forma correlativa con aquella recuperación y, por ende, las posiciones de los jóvenes en este están estructuradas por la persistencia del desempleo juvenil, la baja calidad del empleo y un nivel de precariedad que ronda el 40 por ciento (Jacinto, 2010).

El trabajo de Pérez, Deleo y Fernández Massi (2013), sostiene la persistencia de las desiguales posibilidades que poseen los jóvenes al momento de insertarse en el mundo del trabajo. En este sentido, la mejoras en las condiciones laborales presentes en los tres estratos 
-bajo, medio y alto- no implicó una reducción significativa de la desigualdad que existe entre ellos. Las desigualdades vinculadas al origen social ponen en escena la importancia de recuperar la dimensión estructural como factor explicativo y no enfatizar exclusivamente el nivel educativo.

Por otra parte, adquiere importancia la segmentación del mercado de trabajo como variable para comprender la inserción de los jóvenes y las demandas que generan los distintos sectores productivos. Los sectores de construcción y servicio doméstico son los que generan empleos de menor calidad y, como veremos a continuación, son aquellos en los que se encuentran insertos gran parte de los jóvenes estudiantes del Plan FinEs2. En cambio, en el sector de la actividad manufactura, transporte y comunicaciones existe una tendencia de mayor calidad en los empleos (Salvia y Vera, 2013). Es decir, dar cuenta de la segmentación del mercado de trabajo y del nivel de calidad y formalidad de los empleos que cada segmento crea, constituyen factores a tener en cuenta al momento de indagar los procesos de inserción laboral de jóvenes. Por último, si bien entendemos a la(s) juventud(es) en plural debido a las grandes heterogeneidades que cruzan las experiencias de ser joven, a edades tempranas las experiencias laborales no presentan grandes variaciones. Es decir, en un primer momento de la trayectoria laboral la condición juvenil puede explicar las condiciones de precariedad. Avanzada dicha trayectoria, la educación y el estatus socioeconómico adquiere mayor importancia explicativa al momento de abordar las condiciones laborales de los jóvenes (Saraví, 2009). Las diferenciaciones entre las condiciones de empleo se tornan mayores y, por ende, la consolidación de las desigualdades. En el caso de los jóvenes de sectores populares, las inserciones laborales de tipo precarias pueden ser definitivas viéndose excluida la posibilidad de acceder a trabajos estables. De esta forma, las credenciales educativas, la segregación espacial y otras dimensiones de la desigualdad social son de carácter acumulativas al formar parte de los procesos de acumulación de desventaja (Saraví, 2009).

\section{La aplicación de las nociones de empleo y trabajo como forma de análisis}

En el presente apartado abordaremos los procesos de inserción laboral de los jóvenes entrevistados bajo la idea de dinámicas desiguales. Específicamente, avanzaremos en el estudio de la configuración de posiciones objetivas en el mercado de trabajo y en la comprensión de las producciones subjetivas que los jóvenes realizan sobre los procesos de inserción.

Si bien el artículo no tiene como objetivo central estudiar exhaustivamente las condiciones de precariedad en el mundo laboral, constituirá un elemento analítico a retomar. Específicamente, recuperamos la distinción entre "precariedad del empleo" y "precariedad del trabajo" que realiza 
Paugam (2000) con el objetivo de dar cuenta de los componentes objetivos y subjetivos presentes en el proceso de inserción laboral, dejando de lado las preocupaciones en torno a las formas de integración laboral que dicho autor aborda.

En relación a estas dos dimensiones hacemos referencia, por un lado, al reconocimiento material y simbólico del trabajo -dimensión subjetiva- y, por el otro lado, a la estabilidad en el empleo y los derechos vinculados a la formalidad-dimensión objetiva-. En palabras de Longo:

Paugam se referirá entonces al 'empleo precario' en situaciones laborales donde, para el trabajador, su empleo es incierto y no puede prever su futuro profesional. Esta situación, se caracteriza por una fuerte vulnerabilidad económica y por una restricción, al menos potencial, de los derechos sociales. Pero por otro lado, hablará de 'trabajo precario' cuando el trabajador no tiene un debido reconocimiento material y simbólico por su actividad (2014: 43).

\section{Inserciones laborales: un análisis de la dimensión objetiva}

A continuación se recuperará como nivel de análisis las posiciones de la familia y sus condiciones en el mercado de trabajo para, luego, profundizar las relaciones entre origen social y mercado de trabajo.

Si bien las trayectorias laborales no son fijas ni estables, cuando analizamos las relaciones que se establecen con el mundo del trabajo encontramos ciertas características comunes. Los integrantes de las familias de los jóvenes estudiantes han ingresado al mercado de trabajo desde temprana edad y en actividades informales, principalmente en los sectores de construcción y de servicio doméstico. A su vez, las experiencias laborales de los adultos referentes presentan como característica general la informalidad y la ausencia de derechos laborales.

Sin intención de construir argumentaciones lineales, es posible afirmar que las experiencias de los jóvenes presentan ciertas similitudes. En el caso de Catriel (1), estudiante del segundo año del FinEs2 de 17 años, el relato sobre sus primeros trabajos es organizado a partir de las experiencias laborales de su padre. Este, si bien se encuentra trabajando en una dirección de cooperativas que dependen de la Municipalidad de La Plata, anteriormente trabajó de albañil, electricista y mecánico. Catriel ingresa al mercado de trabajo a los siete años de edad y su recorrido laboral se construye en vinculación con las inserciones laborales de su padre y hermano: peón de albañil, pintor, mecánico y electricista.

Este elemento es algo que está presente en distintas entrevistas, ya que las estrategias de los jóvenes para alcanzar los primeros empleos están vinculadas a los trabajos de los integrantes de sus grupos de pertenencia y al despliegue de redes de relaciones relativamente cercanas. Salvo Catriel, quien empezó a trabajar a los siete años, el resto de los jóvenes entrevistados 
obtuvieron sus primeros empleos a los trece o catorce años. De esta forma, las experiencias laborales se encuentran influenciadas por las posiciones sociales familiares. Las fuertes necesidades económicas y la temprana conformación de sus propias familias son elementos constantes en los distintos relatos.

$\mathrm{P}:$ ¿Y tus viejos trabajan?

R: Mi papá (...) Mi papá era repartidor de diario, que también un poco... por él yo trabajaba los fines de semana. Repartía el diario...

P: ¿Desde qué edad empezaste a trabajar?

R: Catorce más o menos... primero desde los trece, catorce, mi hermana me había dado una casa cerca que iba una vez cada tanto, cada quince días, una cosa así. Después de los catorce vendía diarios, pero los fines de semana nada más porque los días de semana iba al colegio. Después dejé, no trabajé hasta los quince más o menos (Marianela, estudiante de tercer año del FinEs2, 23 años).

Con el objetivo de comprender las posiciones de los jóvenes en el mercado de trabajo, proponemos delinear algunas de las condiciones comunes que encontramos en los procesos de inserción laboral.

En primer lugar, los ingresos tempranos de los jóvenes entrevistados se explican por las necesidades económicas de las familias cuyos ingresos no eran suficientes. De esta manera, las dificultades en complementar tareas laborales y educativas y las prácticas selectivas de las instituciones escolares del nivel secundario, con una fuerte tradición excluyente, constituyeron algunos de los factores que influyeron en el retiro no deseado del sistema educativo.

En estos casos, la entrada al mundo laboral sin haber finalizado la educación secundaria constituye una de las dimensiones de las dinámicas desiguales. Estas operan en el proceso de inserción laboral y en la acumulación de desventajas que hacen a la configuración de las posiciones de estos jóvenes en la estructura social (Saraví, 2009). Según un estudio que realiza Pérez (2011) retomando datos la Encuesta permanentes de hogares (EPH) de 2003, el $49.26 \%$ de los jóvenes de sectores populares no completaron estudios secundarios. En palabras del autor:

\footnotetext{
uno de cada dos jóvenes de entre 19 y 24 años de clase obrera abandonan el sistema educativo antes de terminar el colegio secundario, en muchos casos producto de los bajos ingresos del hogar, que obliga a adelantar la salida de los jóvenes al mundo del trabajo, aun antes de concluir su formación. Esta proporción disminuye a 18.8\% para la clase media y a 15\% para la clase alta (Pérez, 2011: 147).
}

En el desarrollo de las trayectorias laborales de los jóvenes, sólo una de las estudiantes logró obtener un trabajo estable en una empresa de servicios domésticos. El resto, se encuentra 
trabajando en cooperativas que dependen de la Municipalidad de La Plata o de la provincia de Buenos Aires y en otros rubros del sector servicios, bajo contrataciones informales y precarias.

P: Contame un poco qué implica trabajar en la cooperativa...

R: No, está bueno, pero pasa que llega un momento que te cansa y por ahí nosotros estamos en la parte de zanjeo y zanjeamos... no tenés tiempo de descansar porque tenemos que trabajar ahí (...) No aguantas... a veces llegas a tu casa, te agarra con todo esto, tenés que salir a volantear, no descansas, no estás en tu casa.

$P$ : ¿Y cómo son las condiciones (...) tenés una obra social?

R: No, no... es como si laburo en negro, sí, porque no tenemos obra social, no tenemos nada. Así que... y nos pagan por mes que no... no es mucho pero tampoco es poco. Es justo, o sea, te conformas, te acostumbras a cobrar lo que tenés y te conformas con lo que te dan... (Yessica, estudiante de tercer año del FinEs2, 24 años).

La informalidad es la segunda característica de los procesos de inserción que es compartida. Esta es entendida como un conjunto de relaciones sociales que se alejan del típico vínculo salarial y comprenden un conjunto amplio y heterogéneo de actividades "...donde las condiciones de trabajo resultan poco seguras; los ingresos de los trabajadores se encuentran por debajo de los formales; el acceso a la protección social es deficiente y la explotación y la violación de los derechos de los trabajadores son prácticas habituales" (Pérez, 2011: 143). El peso de las dinámicas desiguales que estructuran el mercado de trabajo argentino y el lugar de los jóvenes de sectores populares en él explican, en parte, el proceso de configuración de las posiciones objetivas en el escenario laboral y las condiciones bajo las cuales estos se insertan. Por último, otra de las características presente en las trayectorias laborales de los jóvenes son las múltiples transiciones entre estudio, trabajo e inactividad. La multiplicidad de recorridos y posiciones nos permite dar cuenta de las secuencias de inserciones presentes en los procesos de entrada al mundo laboral (Longo, 2011). La individualización y la diferenciación de las trayectorias de los jóvenes se explican por la variedad de factores que intervienen, desde niveles macroeconómicos hasta individuales o familiares. Son los casos de las últimas entrevistas realizadas en 2015 , donde las jóvenes mujeres no se encontraban trabajando ni buscando trabajo debido a acontecimientos familiares como los nacimientos de sus hijos. En ese momento se dedicaban, exclusivamente, a la formación y al desarrollo de trabajos domésticos y de crianza.

En los primeros recorridos de los jóvenes, la complementariedad entre trabajo y educación es posible. Luego, las transiciones entre estudio, empleo e inactividad adquieren protagonismo en los procesos de inserción laboral debido a distintos factores como la distribución sexual del trabajo. En el caso de las mujeres predomina el trabajo de la crianza en la constitución de familias propias; en el caso de los hombres, la creciente intensidad de los trabajos realizados. 
Dar cuenta de estas tres condiciones comunes -ingresos tempranos, informalidad y múltiples transiciones entre estudio, trabajo e inactividad- tiene como objetivo presentar algunas de las dimensiones que intervienen en la configuración de las posiciones objetivas de los jóvenes en el mercado de trabajo.

\section{Inserciones laborales: un análisis de la dimensión subjetiva}

Con el objetivo de recuperar la importancia del orden simbólico que está presente en la propuesta conceptual de Paugam (2000), específicamente en la noción de trabajo precario, retomamos el concepto de disposiciones laborales que nos permitirá dar cuenta de las representaciones y sentidos que construyen los jóvenes. Longo (2011) distingue cuatro tipos de disposiciones para estudiar las formas en que los jóvenes entienden el mundo laboral: disposición a la actividad, disposición al trabajo, disposición al empleo y disposición al mundo laboral. Esta noción nos permite abordar las distintas dimensiones que presenta la vida laboral para comprender la multiplicidad de posicionamientos de los individuos (Longo, 2014).

En primer lugar, la disposición a la actividad hace referencia a la importancia que presenta el trabajo en la vida de los jóvenes. En segundo lugar, la disposición al trabajo da cuenta de las razones y motivaciones de aquellas primeras vinculaciones con el mundo laboral. En tercer lugar, la disposición al empleo se refiere a la importancia del trabajo típico asalariado y los criterios de evaluación de los empleos. Por último, la disposición al mundo laboral se vincula con las percepciones en torno a las condiciones, oportunidades y restricciones que poseen los jóvenes en este. Nos centraremos especialmente en las disposiciones al empleo y al mundo laboral, aunque caracterizaremos brevemente las dos restantes.

Como hemos abordado en el apartado anterior, todos los jóvenes entrevistados ingresaron al mundo del trabajo a edades tempranas debido a las propias dinámicas de las economías domésticas y a los bajos ingresos que las caracterizan. Es por ello que la actividad y la importancia del campo laboral influyen sobre las experiencias de sus juventudes.

De esta forma y en relación a la primera disposición, la actividad laboral constituye una esfera central. En este marco, la decisión de volver a ingresar al mundo educativo se vincula con la oportunidad de finalizar sus estudios en el marco de una política pública de terminalidad educativa -como es el Plan FinEs2- adquiriendo progresiva importancia.

En relación a la importancia otorgada a la actividad, las jóvenes que en su mayoría trabajan en cooperativas y se encuentran en el último tramo de su recorrido educativo, establecen una clara distinción entre lo público y lo privado. La actividad, el trabajo, sin distinciones, implica la importancia de "salir de casa", tener otras obligaciones y responsabilidades alejadas de las actividades domésticas que tradicionalmente son otorgadas a la mujer. Es el caso de Yanina, que en el momento de la entrevista se encontraba inactiva debido al nacimiento reciente de su 
segundo hijo. Ella plantea la necesidad y el deseo de estar estos primeros meses con su hijo pero, a su vez, la importancia de volver a trabajar, de recuperar sus responsabilidades fuera de la casa, es decir, del escenario privado.

Este último aporte nos lleva a preguntarnos por las disposiciones al trabajo. La importancia de la actividad laboral radica en la posibilidad de lograr mejores posiciones económicas y de vida. La constitución de sus propias familias y el proyecto de poseer una casa propia, principalmente de material, constituyeron las dimensiones centrales que los jóvenes hacen referencia al momento de evaluar las motivaciones o las razones de sus primeras inserciones.

En relación a la pregunta de qué significa trabajar, Yanina afirma:

R: Y tener mi plata, poder ayudar a mi marido, viste, seguir progresando en mi casa, que es lo que más queremos nosotros, viste, que no nos falte nada, es la comodidad, para los dos, para los dos nenes... y yo quiero eso terminar mi casa bien terminada como se debe y... tener todas las comodidades (Yanina, estudiante del tercer año del FinEs2, 24 años).

Por otro lado, las disposiciones al empleo tienen estrecha vinculación con la idea de "buen empleo" o "mejor trabajo" presente en los relatos de los jóvenes. La búsqueda de un "mejor trabajo" se encuentra en los principales motivos que estuvieron presentes en la decisión de volver a estudiar y en los criterios para evaluar los distintos empleos. Frente a la pregunta en relación a cómo ellos definen la idea de un buen trabajo, relatan, principalmente, las ventajas de la estabilidad y los beneficios propios de los derechos laborales. Vacaciones, aguinaldo y obra social, constituyen algunos de los elementos presentes en los jóvenes.

R:lo que yo quiero es esto, quiero un trabajo... no importa lo que sea, estoy dispuesta a trabajar de lo que sea pero que sea un trabajo en blanco (...) Y el trabajo en negro cobras siempre menos... y, en cambio, el trabajo en blanco, qué se yo, vos tenés posibilidades... tenés obra social, tenés un montón de cosas que a mí me sirven para mis hijos y... te pagan mejor y vos sabes que es más difícil que te echen porque te tienen que indemnizar (Leticia, estudiante de tercer año del FinEs2, 24 años).

Si bien autores como Castel (2009) y Sennet (2010) afirmaron la progresiva corrosión del trabajo asalariado típico de la sociedad fordista, esta idea sigue teniendo un peso muy fuerte en las producciones simbólicas y en los criterios de los jóvenes entrevistados. De esta forma, el trabajo formal, estable, entendido como trabajo asalariado tracciona en la construcción de deseos y proyectos y opera como organizador de prácticas y proyecciones.

Las disposiciones al empleo tienen estrecha relación con las disposiciones al mundo laboral y con los sentidos otorgados al mundo educativo. Las representaciones en torno al conocimiento y sus titulaciones nos permiten establecer los nexos que los jóvenes construyen entre formación, certificaciones y las oportunidades que se abren en el mercado laboral. 
Las disposiciones al mundo laboral -sus condiciones, oportunidades y restricciones- que los jóvenes construyen están asociadas, principalmente, a una mirada que pone el foco en la falta. Todo aquello que no lograron realizar -como finalizar sus estudios secundarios- es representado como obstáculos para la obtención de un "mejor trabajo". Por ende, consideran como necesario revertir dicha situación para lograr acceder a las condiciones de ingreso al mercado de trabajo y sus posibilidades de movilidad en él.

Teniendo estas significaciones como marco, poseer el título secundario constituye, desde el discurso de los estudiantes, un pasaporte directo al "buen empleo":

P: ¿Tu hermana, que fue la primera que terminó la secundaria, nunca pensó en estudiar otra cosa?

$\mathrm{R}$ : $\mathrm{Si}$, nosotros siempre se lo dijimos. Pero no sé, ella porque terminó el colegio secundario y enseguida tuvo un hijo entonces ella ponía, no sé si excusas (...) y... tampoco sigue trabajando de empleada doméstica... pero yo siempre le digo vos que tenés el secundario podrías buscarte otro trabajo, o cajera en un supermercado, no se cualquier cosa o administrativa... (Marianela, estudiante de tercer año del FinEs2, 23 años).

La relación directa entre trabajo típico asalariado y el cumplimiento de la formación secundaria se tensiona cuando retomamos los trabajos que, desde una perspectiva macro, sostienen que en las conexiones entre formación y acceso al empleo se ponen en juego otras variables como el origen social de la familia (Pérez, 2008; Jacinto, 2010). De esta forma, aquello que aparece como posible en el orden subjetivo, en tanto la educación secundaria es concebida como posibilitadora de movilidad social ascendente, se tensiona cuando las dinámicas sociales de la desigualdad intervienen sobre las equivalencias que anteriormente existían entre nivel de formación y acceso y calidad del empleo (Dubar, 1991).

\section{Un intento de articulación. Posiciones en el mundo del trabajo}

Para analizar los procesos de inserción laboral se han abordado las posiciones de los jóvenes estudiantes en el mercado de trabajo, dando cuenta de las producciones simbólicas en torno al mundo laboral. En este apartado, el objetivo es avanzar en la articulación de las dimensiones objetivas y subjetivas, con la intención de identificar tipos de posiciones específicas.

Por un lado, retomaremos las ideas de trabajo informal y "buen empleo" -esta última como categoría nativa- para dar cuenta de los componentes objetivos de la inserción laboral. Por otro lado, incorporaremos las producciones simbólicas que los jóvenes atribuyen a sus trabajos bajo la idea de reconocimiento y valoración -negativa y positiva-.

A continuación, presentaremos cuatro tipos de posiciones que funcionan como referentes de las experiencias heterogéneas y de las significaciones de los procesos de inserción laboral. La 
idea es establecer un continuum donde las cuatro posiciones se encuentran configuradas por el cruce de las dos dimensiones.

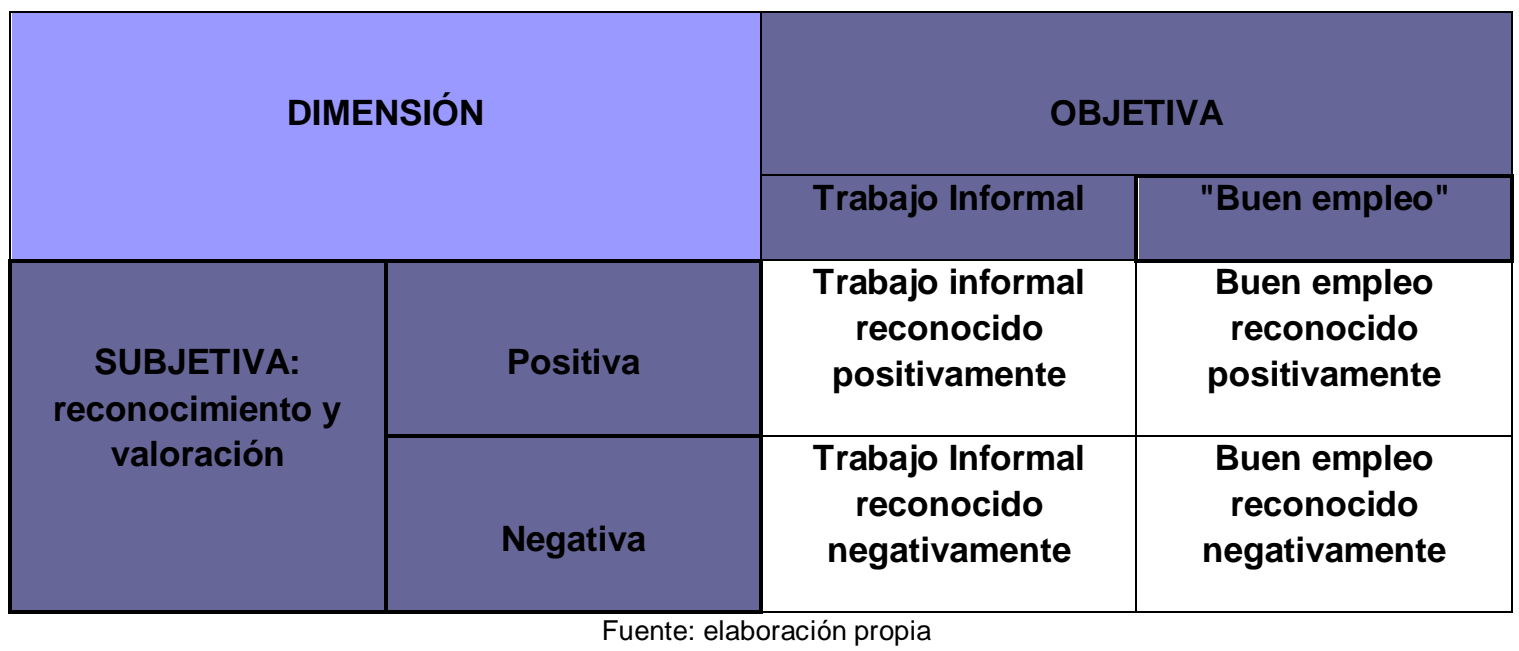

La formalidad no es la dimensión exclusiva por medio de la cual los jóvenes evalúan sus trabajos. Como planteamos al principio del artículo, recuperamos a Paugam (2000) con el sentido de dar cuenta de aquellas producciones simbólicas relacionadas con valoraciones positivas y negativas. Desde trabajos informales, que presentan otros tipos de ventajas que habilitan a reconocimientos positivos hasta trabajos registrados, cercanos a la típica relación asalariada, que pueden ser avaluados negativamente por los jóvenes. Con el objetivo de aproximarnos a la comprensión de estos cuatro tipos de posiciones en el mundo del trabajo, presentaremos algunas de sus ideas rectoras.

\section{Trabajo informal reconocido positivamente}

Pamela, al igual que muchas de las jóvenes mujeres entrevistadas, se encuentra inserta en cooperativas de trabajo que dependen de la Municipalidad de La Plata o la provincia de Buenos Aires. Sus posiciones en el mundo laboral se encuentran alejadas de las relaciones salariales típicas, estructuradas por una ausencia de la protección laboral y los derechos asociados. A su vez, las exigencias físicas del trabajo, propias de la actividad del zanjeo, se entrelazan con actividades políticas de la organización social que coordinan las cooperativas, las cuales ellas mismas consideran parte de las responsabilidades y obligaciones laborales.

Trabajos que, si bien poseen condiciones informales y precarias, pueden presentar otro tipo de ventajas: mejores horarios, mayor comodidad, posibilidad de aprendizajes, entre otros. A su vez, las múltiples inserciones que han tenido los jóvenes entrevistados -siempre bajo condiciones informales- posibilita la construcción de criterios de evaluación que exceden las condiciones objetivas. 
Este es el caso de Pamela, que se encuentra trabajando en una cooperativa y cumple tareas en una oficina administrativa municipal de reclamos de barrido y limpieza:

R: ... me encargo de los reclamos de los cero ochocientos y también estoy para el barrio donde me encargo...

P: ¿Y te gusta el laburo en la oficina?

R: Sí, sí, es otra cosa (...) porque vas, vas... estoy aprendiendo cosas que antes no hacía, o sea, computadora cero y ahora estoy continuamente con computadoras, eso es una ayuda también más... que me va ayudar para el día de mañana (...) pero no, está bueno, aprendí más cosas y conoces más gente, estás con políticos, con concejales, está bueno (Pamela, estudiante del segundo año del FinEs2, 24 años).

Las valoraciones positivas no excluyen el reconocimiento de las condiciones objetivas, hecho que permite proyectar la búsqueda de otro trabajo pero con la valoración actual de mejoras salariales y de aprendizajes vinculados a las tareas diarias.

\section{Trabajo informal reconocido negativamente}

Si en el caso anterior podemos sostener que existen tensiones entre las condiciones objetivas -trabajo informal- y las valoraciones positivas, en este la correspondencia entre ambas dimensiones es más fuerte: trabajo informal que es reconocido negativamente. Este segundo tipo de posición es la más representativa en términos de las experiencias y las significaciones de nuestra muestra.

Es posible retomar el caso de Lorena y Catriel por tener distintas inserciones bajo similares condiciones: la primera en una cooperativa de trabajo y el segundo como ayudante de albañil.

P: ¿Cómo sería ese mejor trabajo? ¿De qué te gustaría laburar?

E: Un trabajo fijo, tener un horario de tal horario a tal horario. Algo que me paguen en blanco, tener todo. Más que nada tener un horario. Porque acá es como ya te dije... tenés que venir y tenés que estar a disposición, es así...

P: Claro, ¿ustedes hacen todo el trabajo manual?

E: Sí, sí, de lunes a viernes estamos ahí en el zanjeo (...) y después... si nos toca repartir volantes, repartimos volantes o si nos toca hablar con la gente, hablamos con la gente (Lorena, estudiante del tercer año del FinEs2, 24 años).

P: ¿Ahora estás buscando un mejor trabajo?

E: No, el que tengo por ahora puede tirar y después cuando consiga un buen laburo voy a seguir trabajando y estudiando...

P: ¿Para vos qué sería un buen laburo? 
E: Un buen sueldo por mes, que no te cortes laburando, todas esas cosas así... qué se yo... que no te mates laburando, haciendo cosas pero no tanto (...) Albañil es lo que más laburo, es lo que ahora puedo conseguir es albañil, entrar piedras y todas esas cosas... lo que te mata es la cintura (Catriel, estudiante del primer año del FinEs2, 17 años).

A partir de la idea de "buen trabajo" muchos de los jóvenes daban cuenta de los sentidos en torno a sus posiciones en el mundo laboral. En ambos casos el esfuerzo físico y la carga horaria destinada a sus trabajos contribuyen a desplegar valoraciones negativas. A su vez, las mujeres entrevistadas que se encuentran trabajando en las cooperativas plantean la necesidad de tener un horario relativamente fijo. La importante carga horaria de sus tareas diarias, otorgadas a obligaciones vinculadas a actividades políticas y de militancia territorial, generan situaciones de reconocimientos negativos.

\section{"Buen empleo" reconocido negativamente}

Marianela es uno de los pocos casos que se encuentra trabajando formalmente en una empresa de limpieza y mantenimiento de edificios. Sin embargo, encontramos una valoración negativa que complejiza la idea de "buen trabajo". De esta manera, refuerza la hipótesis de la necesidad de incluir el análisis de las producciones simbólicas al momento de estudiar los procesos de configuración de las posiciones de los jóvenes.

\section{P: ¿Y has pensado otras posibilidades?}

R: Otras posibilidades... sí, por el momento voy a buscar la manera más rápida de llegar a un trabajo mejor y después ver algún estudio que realmente me guste.

$P$ : ¿Que sería un trabajo mejor?

R: Eh... mejor del que tengo ahora, más cómodo, no sé... menos horarios, menos tiempo (...) una amiga de mi marido nos había contado que (...) en un año ya podes ser preceptora, entonces yo decía bueno ir por ahí que son menos horas, tenemos vacaciones de verano, vacaciones de invierno que ahora no tenemos y esas cosas... más tiempo (...) buscarme un trabajo mejor en el sentido que no me ocupe tantas horas y que, a la vez, me permita estudiar" (Marianela, estudiante de tercer año del FinEs2, 23 años).

La carga horaria, el tipo de trabajo y el esfuerzo físico son algunas de las dimensiones que estructuran las representaciones. Como en el primer referente, encontramos tensiones y poca correspondencia entre las dimensiones objetivas y subjetivas -empleo registrado valorado negativamente-.

"Buen empleo" reconocido positivamente: el imaginario compartido

En las distintas entrevistas realizadas y charlas informales con otros jóvenes estudiantes, no hemos encontrado experiencias que se relacionen con este último tipo de posición. Igualmente, 
consideramos importante incorporarlo por formar parte de un imaginario compartido que constituye un referente en las proyecciones en torno a lo laboral y sus relaciones con el mundo de lo educativo.

$P:$ ¿Y qué sería para vos un buen trabajo?

R: Y algo así... que puedas tener recibo de sueldo, que tengas obra social, de donde... donde no sea tan así, esclavizado como en la cooperativa (...) porque viste que en la cooperativa vos tenés que cumplir, ir a las movilizaciones, ir a las reuniones (...) todo eso (Yanina, estudiante del tercer año del FinEs2, 24 años).

El mundo de la educación ingresa como espacio central en el proceso de finalizar la formación secundaria para así alcanzar el título y, en la medida de lo posible, continuar los estudios en el nivel superior o en la formación de oficios. Estos proyectos funcionan, desde el discurso de los jóvenes, como pasaporte para acceder a lo que se ha denominado como trabajo típico asalariado (Busso, 2013). En este caso, la idea de "buen empleo" o "mejor trabajo" interviene como criterio de evaluación que permite construir reconocimientos y valoraciones positivas o negativas.

\section{Algunas líneas finales}

Teniendo como marco el interrogante en torno a la configuración de los procesos de inserción laboral de jóvenes estudiantes del Plan FinEs2, en este artículo se han abordado las formas en que se delinean las posiciones de los jóvenes en el mercado de trabajo y las disposiciones sobre las distintas esferas del mundo laboral.

Recuperando la distinción conceptual entre empleo y trabajo, se avanzó en la articulación de las dimensiones objetivas y subjetivas que intervienen en las inserciones laborales (Paugam, 2000). Durante el desarrollo del texto se buscó problematizar las nociones de "buen empleo" y "mejor trabajo", dando cuenta que más allá de los aspectos asociados a la formalidad del empleo existe una pluralidad de representaciones, que hacen más complejas las definiciones de la típica relación asalariada.

En este sentido, se avanzó en la articulación de las dimensiones objetivas -relacionadas a la estabilidad y a las condiciones de trabajo- y subjetivas -vinculadas al reconocimiento material y simbólico- con el objetivo de construir cuatro tipos de posiciones en el mercado de trabajo que permitan deconstruir las complejas y heterogéneas experiencias de los jóvenes y, a su vez, posibilite problematizar las relaciones lineales que se establecen entre nivel de formación, acceso y calidad del empleo. 
Por otro lado, en este artículo hemos considerado que la persistencia de la desigualdad entre jóvenes provenientes de distintas sectores socio-económicos, exige recuperar la problemática de las inserciones laborales. De esta manera, la idea de dinámicas desiguales hace referencia a la necesidad de construir interrogantes que pongan en escena la configuración desigual de las posiciones de los jóvenes en el mercado de trabajo y en otros escenarios de la vida social. Por último, consideramos importante mencionar una tensión encontrada que es necesario profundizar en escritos posteriores. La relaciones lineales entre la formación secundaria y las posibilidades de alcanzar empleos estables y seguros se encuentran presentes en las representaciones que los jóvenes tienen sobre el proceso de "volver a la escuela" en el marco del FinEs2. Sin embargo, la reconstrucción de algunas de las dinámicas que estructuran el mercado de trabajo argentino nos permite sostener que en los vínculos entre educación y empleo se ponen en juego otras variables estructurales como el origen social. De esta forma, aquello que desde lo educativo aparece como posibilitador de la movilidad social ascendente se tensiona cuando las dinámicas sociales de la desigualdad intervienen sobre las equivalencias entre nivel de formación y empleo (Dubar, 1991). En este sentido, y para finalizar, remarcamos la necesidad de estudiar dispositivos que, enmarcados en políticas públicas, intervienen en las trayectorias biográficas de los jóvenes con el objetivo de modificar círculos excluyentes (Jacinto, 2010).

\section{Notas}

(1) Los nombres de los jóvenes entrevistados han sido cambiados para resguardar sus identidades.

\section{Bibliografía}

Battistini, O. y Mauger, G. (2012). La difícil inserción de los jóvenes de clases populares en Argentina y Francia. Buenos Aires, Argentina: Prometeo.

Busso, M. (2013) Precariedad laboral en Democracia (y la persistencia de la estabilidad laboral como norma social, también entre los jóvenes). Revista Cuestiones de sociología de la Facultad de Humanidades y Ciencias de la Educación, 9, pp. 193-196.

Busso, M.; Longo, M. E. y Pérez, P. (2011). Trayectorias socio-ocupacionales de jóvenes argentinos. Un estudio cuali y cuantitativo de procesos de precariedad laboral. En $10^{\circ}$ Congreso Nacional de Estudios del Trabajo. Pensar un mejor trabajo. Acuerdos, controversias y propuestas. Asociación Argentina de Especialistas en Estudios del Trabajo, Buenos Aires. 
Dubar, C. (1991). La socialisation. Construction des identités sociales et professionnelles. Francia, París: Armand Colin Editeur.

Jacinto, C. (comp.) (2010). La construcción de las trayectorias laborales de jóvenes. Políticas, instituciones, dispositivos y subjetividades. Argentina, Buenos Aires: Editorial Teseo.

Kessler, G. (2014). Controversias sobre la desigualdad. Argentina, 2003-2013. Argentina, Buenos Aires: Fondo de Cultura Económica, Buenos Aires.

Longo, J. (2014). ¿Renovación de las tradiciones sindicales en ámbitos laborales precarizados? Un análisis de las organizaciones sindicales en empresas supermercadistas durante la posconvertibilidad (tesis de doctorado). Universidad de Buenos Aires, Buenos Aires.

Longo, M. E. (2011). Trayectorias laborales de jóvenes en Argentina. Un estudio longitudinal de las prácticas de trabajo, las disposiciones laborales y las temporalidades juveniles de jóvenes de la Zona Norte del Gran Buenos Aires, en un contexto histórico de diferenciación de las trayectorias (tesis de doctorado). Universidad de Buenos Aires, Buenos Aires.

Paugam, S. (2000). Le salarié de la précarité. Francia, París: PUF.

Pérez, P. (2008). La inserción ocupacional de los jóvenes en un contexto de desempleo masivo. El caso argentino entre 1995 y 2003. Argentina, Buenos Aires: Miño y Davila Editores/Ceil-Piette CONICET.

Perez, P. (2011). Jóvenes, estratificación social y oportunidades laborales. Revista Lavboratorio, 24, pp. 134-153.

Pérez, P.; Deleo, C. y Fernández Massi, M. (2013). Desigualdades sociales en trayectorias laborales de jóvenes en la Argentina. Revista Latinoamericana de Población, 7(13), pp. 61-89.

Salvia, A. y Vera, J. (2013). Heterogeneidad estructural, calidad de los empleos y niveles educativos de la fuerza de trabajo en la Argentina post reformas estructurales (20042007-2011). En $11^{\circ}$ Congreso Nacional de Estudios del Trabajo. El mundo del trabajo en discusión. Avances y temas pendientes. Asociación Argentina de Especialistas en Estudios del Trabajo, Buenos Aires.

Saraví, G. (2009). Transiciones vulnerables. Juventud, desigualdad y exclusión en México. México, Ciudad de México: Publicaciones de la Casa Chata. 